Background/aims We developed a novel, long-term conditionspecific Interactive Health Communication Application, the online parent information and support (OPIS) to promote parents' home-based management ability. We aimed to assess feasibility of a future full-scale randomised clinical trial (RCT) of OPIS in terms of recruitment, retention and data collection procedures; and investigate trends in change on outcomes in a small-scale preliminary RCT in parents.

Methods Parents were randomly assigned to: usual support (control) or usual support plus OPIS access for 20 weeks (intervention). Both groups completed study measures at study entry (T1) and exit (T2). We assessed feasibility between groups.

Results 55 parents of 39 children enrolled in the RCT;19/26 $(73 \%)$ of intervention parents and 22/29 (76\%) of control parents completed T2 data collection. The overall retention rate was 41/55 (75\%). Data collection was judged to be feasible. All intervention parents showed evidence of having accessed OPIS, indicating complete uptake. The intent-to-treat analysis showed greater improvement in self-efficacy to manage their child's condition for intervention parents when compared to control group parents $(3.21$ v $1.09,95 \%$ CI -1.27 to 5.51 , Cohen's $\mathrm{d}=.41)$.

Conclusion A full-scale RCT of OPIS is feasible. OPIS has the potential to beneficially affect self-reported outcomes including parents' competence to provide home-based clinical care-givin. ll-scale RCT that is sufficiently powered to detect the effects of OPIS on outcomes is indicated. Our design and methodology could potentially be transferred to the management of other conditions.

Acknowledgements Families and professionals who participated and the National Institute of Health Research for funding support.

\section{0-220 EXCESSIVE GROWTH FROM 6 TO 24 MONTHS OF AGE: RESULTS FROM THE PREVENTION OF OVERWEIGHT IN INFANCY (POI) RANDOMISED CONTROLLED TRIAL}

${ }^{1}$ SL Cameron, ${ }^{2} \mathrm{AR}$ Gray, ${ }^{3}$ RW Taylor, ${ }^{1} \mathrm{~J}$ A Lawrence, ${ }^{1} \mathrm{BC}$ Galland, ${ }^{1} \mathrm{MB}$ Hanna, ${ }^{4} \mathrm{ALM}$ Heath, ${ }^{1}$ RM Sayers, 'BJ Taylor. 'Women's and Children's Health, University of Otago, Dunedin, New Zealand; 'Preventive and Social Medicine, University of Otago, Dunedin, New Zealand; ${ }^{3}$ Medicine, University of Otago, Dunedin, New Zealand; ${ }^{4}$ Human Nutrition, University of Otago, Dunedin, New Zealand

\subsection{6/archdischild-2014-307384.290}

The Prevention of Overweight in Infancy study investigated whether education and support around sleep, physical activity, and diet could reduce excessive weight gain in the first two years of life. The analysis presented here assessed weight at 24 months postpartum and growth from 6 to 24 months. 802 mother-infant pairs were randomised to: 1) FAB - food, activity and breastfeeding $(8+$ contacts $), 2)$ Sleep - prevention and treatment of sleep problems $(2+$ contacts $), 3)$ Combo - both interventions $(10+$ contacts), or 4) Control. All groups received standard government funded "Well Child" care (7 contacts). Anthropometric measurements were obtained at 6, 12, 18 and 24 months postpartum by trained measurers blinded to group allocation. Rapid and extremely rapid growth were defined as a change in BMI zscore $>2 / 3$ SD (World Health Organisation definition) and $>4$ / 3 SD respectively. $84.5 \%(n=678 / 802)$ of participants were followed up at 24 months with $40.1 \%$ having BMIs $\geq 85$ th percentile $(\mathrm{n}=272)$. Among those with both 6 and 24 month data, $53.9 \%(351 / 651)$ showed rapid growth with 148 of these showing extremely rapid growth $(22.7 \%$ of the sample). There was no difference between intervention groups for rapid growth $(\mathrm{p}=0.892)$ or extremely rapid growth $(\mathrm{p}=0.630)$ compared to normal growth. Similarly, there was no intervention effect on those classified as overweight at 24 months who also displayed rapid or extremely rapid growth $(\mathrm{p}=0.936$ for rapid growth, $\mathrm{p}$ $=0.485$ for excessive rapid growth) from 6-24 months. Our results indicate it is difficult to modify excessive growth.

\section{0-221 SAFEBOOSC - A PHASE II RANDOMISED CLINICAL TRIAL ON CEREBRAL NEAR-INFRARED SPECTROSCOPY OXIMETRY IN EXTREMELY PRETERM INFANTS}

${ }^{1} \mathrm{~S}$ Hyttel-Sorensen, ${ }^{2} \mathrm{~A}$ Pellicer, ${ }^{3} \mathrm{~T}$ Alderliesten, ${ }^{4} \mathrm{~T}$ Austin, ${ }^{3} \mathrm{~F}$ van Bel, ${ }^{3} \mathrm{M}$ Benders, ${ }^{5} \mathrm{O}$ Claris, ${ }^{6}$ E Dempsey, ${ }^{7} \mathrm{AR}$ Franz, ${ }^{8} \mathrm{M}$ Fumagalli, ${ }^{9} \mathrm{C}$ Gluud, ${ }^{9} \mathrm{~B}$ Grevstad, ${ }^{10} \mathrm{C}$ Hagmann, ${ }^{3} \mathrm{P}$ Lemmers, ${ }^{11} \mathrm{~W}$ van Oeveren, ${ }^{12} \mathrm{G}$ Pichler, ${ }^{1} \mathrm{AM}$ Plomgaard, ${ }^{2} \mathrm{~J}$ Riera, ${ }^{2} \mathrm{~L}$ Sanchez, ${ }^{9} \mathrm{P}$ Winkel, ${ }^{13} \mathrm{M}$ Wolf, ${ }^{1} \mathrm{G}$ Greisen. ${ }^{1}$ Neonatology, Rigshospitalet Copenhagen University Hospital, Copenhagen, Denmark; ${ }^{2}$ Neonatology, La Paz University Hospital, Madrid, Spain; ${ }^{3}$ Neonatology, University Medical Center Utrecht Wilhelmina Children's Hospita, Utrecht, Netherlands, ${ }^{4}$ Neonatology, Rosie Hospital Cambridge University Hospitals NHS Foundation Trust, Cambridge, UK; ${ }^{5}$ Neonatology, Hopital Femme Mere Enfants, Lyon, France; ${ }^{6}$ Neonatology, Cork University Maternity Hospital, Cork, Ireland; ${ }^{7}$ Neonatology, University Children's Hospital Tübingen, Tübingen, Germany; ${ }^{8}$ Neonatology, Fondazione IRCCS Ca' Granda Ospedale Maggiore Policlinico, Milano, Italy; ${ }^{9}$ The Copenhagen Trial Unit Centre for Clinical Intervention Research, Rigshospitalet Copenhagen University Hospital, Copenhagen, Denmark; ${ }^{10}$ Neonatology, University Hospital Zurich, Zurich, Switzerland; ${ }^{11}$ Haemoscan BV, Haemoscan BV, Groningen, Netherlands; ${ }^{12}$ Neonatology, Medical University Graz, Graz, Austria; ${ }^{13}$ Biomedical Optics Research Laboratory, University Hospital Zurich, Zurich, Switzerland

\subsection{6/archdischild-2014-307384.291}

Background and aims Extremely preterm infants have a high risk of moderate to severe long-term neurodevelopmental impairment. Hypoxic or hyperoxic brain injury may be a contributing factor. The SafeBoosC trial investigated if it is possible to stabilise the cerebral oxygenation of extremely preterm infants. Methods This was a phase II randomised, single blinded, clinical trial. Infants born before 28 weeks of gestation were eligible. Within $3 \mathrm{~h}$ of birth, infants were randomly assigned to either cerebral near infrared spectroscopy (NIRS) oxygenation monitoring in combination with a treatment guideline (experimental) or blinded NIRS monitoring with standard care (control). The primary outcome was the area under the curve of the time series of absolute deviation from the cerebral oxygenation target range of $55 \%$ to $85 \%$, expressed in \% hours (the burden of hypoxia and hyperoxia). We hypothesised that there would be more than $50 \%$ reduction in this burden in the experimental group.

Results 166 infants with a median postmenstrual age of 26.4 weeks were enrolled (Table 1). Two infants were withdrawn. 86 infants randomised to the NIRS group had a median burden of hypoxia and hyperoxia of $36.1 \%$ hours (IQR 9.2 to 79.5 )

\begin{tabular}{lll} 
Abstract 0-221 Table 1 & & \\
\hline Characteristics & Experimental $(\mathrm{n}=86)$ & Control $(\mathrm{n}=80)$ \\
\hline Birth weight, median (range), g. & $806(410$ to 1286) & $880(490$ to 1330) \\
Gestational age at birth, median (IQR), wk. & $26.6(25.7$ to 27.4) & $26.8(25.5$ to 27.6) \\
Male sex, $\mathrm{n}(\%)$ & $44(51.2)$ & $34(42.5)$ \\
Multiple births, $\mathrm{n}(\%)$ & $20(23.3)$ & $14(17.5)$ \\
Prenatal steroids, $\mathrm{n}(\%)$ & $58(67.4)$ & $56(70.9)$ \\
Prolonged rupture of membranes, $\mathrm{n}(\%)$ & $26(30.6)$ & $32(40.5)$ \\
Maternal clinical chorioamnionitis, $\mathrm{n}(\%)$ & $6(7.1)$ & $7(9.0)$ \\
Apgar score $\leq 5$ at 5 min, $\mathrm{n}(\%)$ & $15(17.6)$ & $14(17.7)$ \\
Umbilical arterial pH, mean (SD) & $7.33(0.088)$ & $7.31(0.096)$ \\
\hline
\end{tabular}

